\title{
Translocation ecology: the role of ecological sciences in plant translocation
}

\author{
Thomas Abeli $\cdot$ Kingsley Dixon
}

Published online: 10 February 2016

(C) Springer Science+Business Media Dordrecht 2016

\section{Introduction}

Translocations (sensu IUCN 2013) can be effective conservation tools only if they are undertaken with underpinning science to support the actions and outcomes. Translocations are rarely the simple exercise of moving species and/or populations for conservation purposes, the so-called 'gardening approach', rather, behind each translocation there should be a level of pragmatic and focused science to support the actions and to interpret the long-term viability of the action. Godefroid et al. (2011) for example, showed that the success of reintroductions can be at risk with even minor gaps in the knowledge of species biology, habitat requirements, threats, etc., leading to failure.

T. Abeli (更)

Department of Earth and Environmental Sciences, University of Pavia, Via S. Epifanio 14, 27100 Pavia, Italy e-mail: thomas.abeli@unipv.it

\section{K. Dixon}

Department of Environment and Agriculture, Curtin University, Bentley, WA 6012, Australia

K. Dixon

School of Plant Biology, University of Western Australia, Crawley, WA 6009, Australia

K. Dixon

Kings Park and Botanic Garden, West Perth, WA 6005, Australia
In the past decade, guidelines have been developed to improve the success of translocations (e.g. Maschinski et al. 2012; IUCN 2013; Rossi et al. 2013) on the basis of experiential evidence, but much more effort is required as the increase in the number of threatened taxa outstrips translocation knowledge and technical capacity. Reasons for failure are manifold, but a lack of knowledge of the species ecological requirements and the selection of ecologically suitable translocation sites loom large as constraints. A key to reduce this uncertainty is through a more active dialogue on the successes and failures of rare plant translocation and through greater integration of ecology into conservation matters.

This special issue highlights "The role of ecological sciences in plant translocation", assembling 10 articles on different ecological issues relevant to successful translocation. Ecological studies are essential in different phases of a translocation and not just to understand the ecological requirements of a target species. Rare species are often rare because they have life history stages that act as bottlenecks to population growth, they may produce few propagules or propagules may be low in viability or have very narrow conditions for recruitment. All these ecological aspects are relevant in translocation as relevant is the selection of suitable sites for translocations (Bontranger et al. 2014), the identification of inter-specific interactions between a target species and the recipient community (i.e. hybridization, pollinator availability, 
competition; e.g. LaBar et al. 2014), the development of monitoring techniques, and more.

An important contribution to all of these aspects comes from the first article of the issue: a review by Reiter et al. (2016) on 96 orchid translocations which shows that establishment of plant-pollinator interactions and mycorrhizal relationships are the key challenges for their successful translocation. This is seminal information as orchid translocations in the past have focused on propagation technologies to the detriment of the long-term viability of the translocated plants.

The challenge of re-establishing working relationships between species is also faced in the article provided by Holzapfel et al. (2016) that analyzes the effect of different hosts on reintroduction success for the holoparasitic plant, Dactylanthus taylorii Hook. This is one of the very few translocation attempts of a holoparasitic plant and highlights that host preference may have important implications in a reintroduction program, providing guiding principles relevant to other parasitic rare plants.

Burney and Burney (2016) present their unique and impressive approach to restore an entire habitat in Hawaii: it is a novel example of community restoration, for more than 80 plant species, based on paleoecological data used to identify the composition of the native flora before the invasion of several alien species. This study shows how historical ecological data can play an important role in understanding reassembly rules in rare plant translocations.

When few data are available on a target species, experimental reintroductions can help to identify the ecological niche of a species and identify a suitable translocation site. This approach was used by Wendelberger and Maschinski (2016) who underline the importance of heterogeneous microsites to increase the probability of success in translocation attempts. Such an approach also has benefits in climate-proofing rare flora translocations.

Godefroid et al. (2016) show through their investigations of four rare European plants the importance of pre-translocation ecological studies aimed at designing suitable translocation protocols using a four-step approach. Moreover, the authors suggest that when material for plant propagation is poorly available, herbaria and seed banks can be important sources of translocation materials.
Similarly, Nakahama et al. (2016) show how ex situ conserved material (specifically herbarium specimens) of rare species can contain unique genetic characteristics that may be missing from extant wild populations. So, if seeds/spores from herbarium vouchers are viable, they can constitute rare opportunity to recover lost genetic diversity of a target species.

Abeli et al. (2016) in their reintroduction and monitoring of a population of the rare European geophyte, Leucojum aestivum L. showed that the study of natural populations of a target species and their reproductive capability, is an effective means to recreate population dynamics that will achieve successful reintroduction.

Also working on a related geophytic species and using a similar approach, Draper et al. (2016) achieved a successful translocation (thirteen-years monitoring), of the rare endemic, Narcissus cavanillesii Barra and López. Faced with the urgency of developing an effective conservation plan for the species due to the construction of a dam, the authors used modeling techniques to identify the climatic envelop where the species could grow and discuss the importance of other ecological information on the success of the translocation.

Fenu et al. (2016) discuss the role of post-translocation management activities on the reintroduction success of the endemic Dianthus morisianus Vals. in Sardinia. In particular, they found that fencing is a simple yet highly effective strategy to reduce the impact of herbivory and humans on translocated populations and to increase the probability of translocation success.

In the final paper of this special issue, Ren et al. (2016), in contrast to most studies, demonstrate that in some species introductions can have the same rate of success as population reinforcements, at least in the short-term.

\section{Conclusion}

Translocations in their many forms (reintroduction, introduction and population reinforcement) have contributed, often in an enduring way, to the conservation of many threatened species. However, for many programs the lack of understanding of the basic ecological capacity of a species within its ecological context means the long-term security of translocated 
populations is at risk. The articles in this special issue focus attention on the way ecological studies can advance translocation ecology as a branch of species conservation science. Understanding plant-pollinator interactions, mychorrizal relationships, and host and microsite preference are all essential components in a translocation, but are also classical ecological determinants of population dynamics and remain fundamental tenants for ensuring long-term success. From this issue we learn how much we know, and don't know, about Translocation Ecology, hence ecological research applied to translocation, identifying where more information is needed.

A final consideration we note as the handling editors for this issue is that we lack literature on where translocations of rare species have failed. Failures provide core diagnostic tools for understanding 'where to next' by way of a post-mortem review. We believe that failures provide guide posts in an 'adaptive management' way that are as important in building future translocation programs as the successes.

\section{References}

Abeli T, Cauzzi P, Rossi G, Adorni M, Vagge I, Parolo G, Orsenigo S (2016) Restoring population structure and dynamics in translocated species: learning from wild populations. Plant Ecol (this issue). doi:10.1007/s11258015-0529-x

Bontrager M, Webster K, Elvin M, Parker IM (2014) The effects of habitat and competitive/facilitative interactions on reintroduction success of the endangered wetland herb, Arenaria paludicola. Plant Ecol 215:467-478. doi:10. 1007/s11258-014-0317-z

Burney DA, Burney Pigott L (2016) Monitoring results from a decade of native plant translocations at Makauwahi Cave Reserve, Kauai. Plant Ecol (this issue). doi:10.1007/ s11258-015-0535-z

Draper DD, Marques I, Iriondo JM (2016) Acquiring baseline information for successful plant translocations when there is no time to lose: the case of the neglected critically endangered Narcissus cavanillesii (Amaryllidaceae). Plant Ecol (this issue). doi:10.1007/s11258-015-0524-2
Fenu G, Cogoni D, Bacchetta G (2016) The role of fencing in the success of threatened plant species translocation. Plant Ecol (this issue). doi:10.1007/s11258-015-0517-1

Godefroid S, Piazza C, Rossi G, Buord S, Stevens A, Aguraiuja $R$ et al (2011) How successful are plant species reintroductions? Biol Conserv 144:672-682. doi:10.1016/j. biocon.2010.10.003

Godefroid S, Le Pajolec S, Van Rossum F (2016) Pre-translocation considerations in rare plant reintroductions: implications for designing protocols. Plant Ecol (this issue). doi:10.1007/s11258-015-0526-0

Holzapfel S, Dodgson J, Rohan M (2016) Successful translocation of the threatened New Zealand root-holoparasite Dactylanthus taylorii (Balanophoraceae). Plant Ecol (this issue).

IUCN (2013) IUCN Guidelines for reintroductions and other conservation translocations. Adopted by SSC Steering Committee at Meeting SC 4,6. 5th September 2012. IUCN Species Survival Commission. IUCN, Gland and Cambridge

LaBar T, Campbell C, Yang S, Albert R, Shea K (2014) Restoration of plant-pollinator interaction networks via species translocation. Theor Ecol 7:209-220. doi:10.1007/ s12080-013-0211-7

Maschinski J, Albrecht MA, Monks L, Haskins KE (2012) Center for plant conservation best reintroduction practice guidelines. In: Haskins KE, Maschinski J (eds) Plant reintroduction in a changing climate. Promises and perils. Island Press, Washington

Nakahama N, Hirasawa Y, Minato T, Hasegawa M, Isagi Y, Shiga $T$ (2016) Recovery of genetic diversity in endangered plants by reintroducing germinated seeds from herbarium specimens. Plant Ecol (this issue)

Reiter NH, Whitfield J, Pollard G, Bedggood W, Argall M, Dixon KW, Davis B, Swarts N (2016) A future for orchid translocations? Plant Ecol (this issue)

Ren H, Wang J, Liu H, Yuan L, Xu Y, Zhang Q, Yu H, Luo J (2016) Conservation introduction resulted in similar reproductive success of Camellia changii compared with augmentation. Plant Ecol (this issue). doi:10.1007/s11258015-0515-3

Rossi G, Amosso C, Orsenigo S, Abeli T (2013) Linee guida per la traslocazione di specie vegetali spontanee. MATTM-Ist. Sup. Protezione e Ricerca Ambientale (ISPRA), Roma

Wndelberger KS, Maschinski J (2016) Assessing microsite and regeneration niche preferences through experimental reintroduction of the rare plant Tephrosia angustissima var. corallicola. Plant Ecol (this issue). doi:10.1007/s11258015-0521-5 